Irene Dingel

\title{
Die Confessio Augustana als Referenzbekenntnis - ihr integratives und abgrenzendes Potenzial
}

In der europäischen Reformationsgeschichte, würde man sie als eine Bekenntnisgeschichte schreiben, kommt der Confessio Augustana ein besonderer Stellenwert zu. Dies gilt noch heute, allerdings unter veränderten historischen Bedingungen und Konstellationen. Dieser Beitrag wird jedoch in die Historie zurückführen, und zwar zunächst zu den Anfängen dieses Bekenntnisses. Sodann soll eine historische Entwicklung skizziert werden, in der die Confessio Augustana zu einem Referenzbekenntnis und damit zum Mittelpunkt eines weitverzweigten theologischen, politischen und rechtlichen Bezugssystems wurde. Referenz ist in unserem Zusammenhang also im Sinne des lateinischen referre, d.h. „sich auf etwas beziehen“, „sich auf etwas zurückführen“ zu verstehen. Die Confessio Augustana wird dabei als ein Dokument in den Blick kommen, das dieses Bezugssystem von der Mitte her konstituiert, und zwar dadurch, dass sich Bekenntnisse unterschiedlicher reformatorischer "Couleur“ auf sie zurückbeziehen und sich auf sie berufen. Der Rückbezug auf die Confessio Augustana bzw. das Selbstverständnis bekenntnisrelevanter Dokumente oder bekenntnishafter Äußerungen als Wiederholung der CA oder als deren Auslegung waren nämlich sowohl in streng lutherisch ausgerichteten Zusammenhängen oder in der Nachfolge Philipp Melanchthons, als auch im reformierten Raum gängig. Dies lässt erkennen, dass dieses Bezugssystem keineswegs konfessionell homogen war. Man konnte, jeweils kontextbezogen, sowohl auf das theologisch integrative als auch auf das bekenntnisgebunden abgrenzende Potenzial der Confessio Augustana rekurrieren. Die Confessio Augustana war also - so die Ausgangshypothese dieses Beitrags noch Jahrzehnte nach ihrer Präsentation und Verlesung auf dem Augsburger Reichstag von 1530 nicht konfessionell festgelegt. Erst langfristig gesehen wurde das Augsburger Bekenntnis, auf dessen Fassung von 1530 - die sogenannte CA invariata - man sich im Zuge der lutherischen Konfessionsbildung in den sechziger und siebziger Jahren des 16. Jahrhunderts wieder zurückbesann, zum Grundsatzbekenntnis des konfessionellen Luthertums. ${ }^{1}$ Aber auch die von Me-

1 Vgl. die Edition der Confessio Augustana durch Volker Leppin, in: Die Bekenntnisschriften der Evangelisch-Lutherischen Kirche (BSELK). Vollständige Neuedition, hg.v. Irene Dingel, Göttingen u. a. $2014,65-225$.

○ OpenAccess. ( 2022 Irene Dingel, publiziert von De Gruyter. (cc) BY-NC-ND Dieses Werk ist lizenziert unter einer Creative Commons Namensnennung - Nicht kommerziell - Keine Bearbeitung 4.0 International Lizenz. https://doi.org/10.1515/9783110683868-008 
lanchthon nach der Wittenberger Konkordie ${ }^{2}$ von 1536 für das Religionsgespräch in Hagenau, Worms und Regensburg überarbeitete Confessio Augustana variata ${ }^{3}$ von 1540, die bis zur lutherischen Konsolidierung in Konkordienformel und Konkordienbuch 1577/1580 im allgemeinen Gebrauch gestanden hatte, erlebte eine späte Renaissance, und zwar im Zuge der konfessionellen Unionen des 19. Jahrhunderts, in deren Zusammenhang sie erneut als innerprotestantisch integratives Element wirken konnte. Dieser spätere Entwicklungsstrang soll aber hier nicht weiter verfolgt werden. Folgendes gilt es vorerst festzuhalten: Ursprünglich war die Confessio Augustana weit davon entfernt als Gründungsdokument einer Konfessionskirche zu dienen. Als Apologia sollte sie vielmehr unter Beweis stellen, dass sich die Evangelischen keineswegs von der Lehre der alten Kirche entfernt hatten. Ja, sie erhob mutig den Anspruch darauf, die Lehre der wahren „katholischen“ Kirche zu vertreten, die sie zugleich von irreführenden Missbräuchen befreit habe und weiterhin befreien wollte. Damit sprach die im Auftrag von Kurfürst Johann dem Beständigen abgefasste Confessio Augustana zunächst nur für Kursachsen, letzten Endes aber für alle im Jahr 1530 und auch später noch hinzukommenden fürstlichen und städtischen Unterzeichner des Bekenntnisses und deren Untertanen.

Im Folgenden soll diese Entwicklung der Confessio Augustana von einem auf Integration zielenden Bekenntnis hin zu einem konfessionell vielfältig herangezogenen Referenzbekenntnis nachgezeichnet werden, wobei auch das konfessionell abgrenzende Potenzial beleuchtet werden muss. Dabei soll zunächst ihre Funktion als Apologia und Defensio im Kontext des Augsburger Reichstags von 1530 zur Sprache kommen, um ihre integrative Zielsetzung zu umreißen. Sodann wird es um jene Entwicklungen gehen, die die Confessio Augustana im Horizont der Repetitio und Interpretatio zu einem Knotenpunkt innerhalb eines theologischen, aber auch politischen und rechtlichen Referenzsystems machten, und schließlich soll die Entwicklung zu konfessioneller Konsolidierung skizziert werden, die sich ebenfalls an der Repetitio und Interpretatio der Confessio Augustana im Spannungsfeld veränderter politischer Bedingungen festmacht.

2 Die Wittenberger Konkordie ist kritisch ediert: Wittenberger Konkordie (1536). Schriften zur Wittenberger Konkordie (1534-1537), bearb. v. Robert Stupperich/Marijn de Kroon/Hartmut Rudolph, Gütersloh 1988 (Bucer-DS 6,1), 114-134.

3 Die Confessio Augustana variata von 1540, bearb v. Volker Leppin, ist ediert in BSELK, Quellen und Materialien (QuM), Bd. 1: Von den altkirchlichen Symbolen bis zu den Katechismen Luthers, hg.v. Irene Dingel, Göttingen u.a. 2014, 119-167. 


\section{Apologia et Defensio - die Confessio Augustana im Kontext des Augsburger Reichstags}

Auf dem von Kaiser Karl V. auf den 8. April 1530 nach Augsburg ausgeschriebenen Reichstag sollte nicht nur die dringende Abwehr der Bedrohung durch die Osmanen beraten werden, die bereits 1529 vor Wien gestanden hatten, sondern es sollten möglichst auch die strittigen Religionsangelegenheiten, die bisher ein politisch effektives außenpolitisches Agieren des Kaisers verhindert hatten, einer Lösung zugeführt werden. Daher war geplant - wie das Ausschreiben lautete -

alle ains yeglichen gutbeduncken: opinion und meynung zwischen uns selbs in liebe und gutligkait zuhoren: zuverstehen: und zuerwegen: die zu ainer ainigen Christlichen warhait zubrengen und zuuergleichen. alles, so zu baide[n] tailen nit recht ist ausgelegt oder gehandelt abzuthun: durch uns alle ain ainige und ware Religion anzunehmen und zuhalten: $[\ldots]^{4}$.

Der sächsische Kurfürst Johann der Beständige veranlasste daher die Wittenberger Theologen, zur Beratung der strittigen Fragen in Lehre und Zeremonien zusammenzukommen und ihr Ergebnis in Torgau vorzulegen. Dies waren möglicherweise die sogenannten Torgauer Artikel, überbracht von Melanchthon am 27. März 1530. Allerdings ist - wie schon Gottfried Seebaß vor Jahrzehnten in einem leider unpublizierten Aufsatz problematisierte - kein Dokument erhalten, das man eindeutig als Torgauer Artikel identifizieren könnte. Dagegen scheint sicher zu sein, dass es verschiedene Stellungnahmen gab, die die Kirchenbräuche zum Gegenstand hatten. ${ }^{5}$ Diese sowie die Schwabacher und die darauf aufbauenden Marburger Artikel Martin Luthers, mit denen im Jahre 1529 das Marburger Religionsgespräch geendet hatte, sind bekanntlich die Grundlagen der Confessio Augustana, deren 1530 präsentierte Fassung nach mehrfacher Umarbeitung durch Melanchthon entstand. Martin Luther, der sich als Geächteter auf der Veste Coburg, dem Augsburg am nächsten gelegenen kursächsischen Grenzort, aufhielt, war an der Abfassung des Bekenntnisses nicht beteiligt, wurde aber brieflich auf dem Laufenden gehalten und erhielt es zur Begutachtung zugeschickt. Seine anerkennende Reaktion, mit der er die Confessio Augustana am 15. Mai 1530 nach

4 Karl Eduard Förstemann (Hg.), Urkundenbuch zu der Geschichte des Reichstages zu Augsburg im Jahre 1530, Bd. 1, Osnabrück 1966 [ND der Ausg. Halle 1833], 8.

5 So Gottfried Seebaß, Die kursächsischen Vorbereitungen auf den Augsburger Reichstag von 1530 - Torgauer Artikel? (unpubliziert). Vgl. auch Volker Leppin, in: BSELK, 66. 
Augsburg zurücksandte, spielt auf den Charakter und die Intention des von kursächsischer Seite vorzulegenden Dokuments an. Es sollte die reformatorische Position zwar klar, aber möglichst unauffällig und diplomatisch darlegen, um Spielräume für eine Akzeptanz der eigenen, reformatorischen Lehre zu schaffen.

Jch hab M. [= Magister] Philipsen Apologia [= das Augsburger Bekenntnis] vberlesen, die gefellet mir fast [= sehr] wol, vnd weis nichts dran zu bessern noch endern, Wurde sich auch nicht schicken, Denn ich so sanfft vnd leise nicht tretten kan. Christus vnser herr helffe, das sie viel und grosse frucht schaffe, wie wir hoffen vnd bitten, Amen! ${ }^{6}$

In der Tat zielte die Confessio Augustana darauf, die Anhänger der römischen Kirche möglichst mit den Positionen der reformatorisch Gesinnten zu versöhnen und die Übereinstimmung der evangelischen mit der wahren „katholischen“ Lehre unter Beweis zu stellen. Zu Recht bezeichnete man sie deshalb als Apologia ${ }^{7}$ und als Defensio ${ }^{8}$. Denn sie trug im Blick auf die römische Kirche weniger abgrenzenden als vielmehr integrativen Charakter, auch wenn dies nicht von allen Seiten so wahrgenommen wurde. Es waren zwei Ziele, die Melanchthon verfolgte: Versöhnen und Bekennen. ${ }^{9}$ Dies versuchte er in Konzeption und Formulierung der einzelnen Artikel geschickt abzubilden, ohne die reformatorischen Kernanliegen zur Disposition zu stellen. So schrieb er zum Beispiel an seinen Freund Joachim Camerarius, dass er die Apologia sehr zurückhaltend abgefasst habe und nicht nachsichtiger hätte sein können. ${ }^{10}$ Er habe bis zum letzten Moment an den For-

6 Luther an Kurfürst Johann, [Veste Coburg], 15. Mai 1530, in: WA Br 5, Nr. 1568, 319,5-9. Diese Bemerkung hat $\mathrm{zu}$ manchen Missverständnissen Anlass gegeben. Unter Bezugnahme darauf wurde Melanchthon nämlich - zu Unrecht - als Leisetreter verunglimpft. Die Bemerkung aber drückt die Bewunderung Luthers für den behutsam und geschickt argumentierenden Freund und Gesinnungsgenossen aus.

7 Vgl. auch Martin Luther an Melanchthon in Augsburg, [Veste Coburg], 29. Juni [1530], in: MBW.T 4,1, Nr. 946, 289,17. Hier schreibt Luther: Accepi apologiam vestram [...]. Vgl. dazu Volker Leppin, Einleitung zur Confessio Augustana, in: BSELK, 65.

8 Vgl. Melanchthon an Martin Luther [auf der Coburg], [Augsburg], 26. Juni [1530]: Caesari est exhibita defensio nostra, quam tibi mitto legendam, in: MBW.T 4,1, Nr. 940, 266,14f.

9 Vgl. Ragnar Andersen, Concordia Ecclesiae. An Inquiry into Tension and Coherence in Philipp Melanchthon's Theology and Efforts for Ecclesiastical Unity, Especially in 1527-1530, Zürich 2016 (Arbeiten zur Historischen und Systematischen Theologie 21), 478-480, bes. 480.

10 Melanchthon an Joachim Camerarius in Nürnberg, [Augsburg], 21. Mai [1530]: Ego apologiam paravi scriptam summa verecundia neque his de rebus dici micius posse arbitror, in: MBW.T 4,1, Nr. 913, 182,16f. 
mulierungen der CA gearbeitet, sei aber keineswegs $\mathrm{zu}$ „weich“ geworden. ${ }^{11} \mathrm{Am}$ 27. Juni 1530, nach der Übergabe der Confessio Augustana an den Kaiser, fragte er auch Luther noch einmal, wie weit man auf die theologischen Gegner zugehen könne: Nostra Confessio est exhibita Imperatori. Exemplum eciam tibi misimus, quod apud te retinebis, ne emanet in publicum. Vetat enim Imperator edere. Scripsi tibi, ut mihi significes, si ita res tulerit, quantum concedere adversariis possimus. ${ }^{12}$ Luther jedoch schien ein noch weiteres Entgegenkommen nicht mehr möglich. Pro mea persona plus satis cessum est in ista apologia, quam si recusent, nihil video, quod amplius cedere possum, nisi videro eorum raciones et scripturas clariores, quam hactenus vidi. ${ }^{13}$ Zugleich betonte Melanchthon gegenüber dem päpstlichen Legaten Lorenzo Campeggio die Friedensbereitschaft der reformatorisch Gesinnten und die dogmatische Übereinstimmung ihrer Lehre mit der der römischen Kirche. ${ }^{14}$ Wie sie bekämpfe man die falsche Lehre, und die Verwerfungen der altkirchlichen Häresien sowie täuferischer Positionen in der Confessio Augustana stellten dies klar unter Beweis. Melanchthon deutete sogar an, dass man ,gegen geringe Zugeständnisse zur Obödienz bereit“ sei. Der doch eigentlich geringfügige Unterschied in Riten und Zeremonien sei „nach dem Kirchenrecht kein Hindernis der Eintracht. “15 Gegenüber Luca Bonfio, dem Sekretär Campeggios, äußerte der Wittenberger die Zuversicht, dass durch das Zugeständnis von Laienkelch,

11 Vgl. Melanchthon an Joachim Camerarius in Nürnberg, [Augsburg], 26. Juni [1530]; hier sein Bericht von der Verlesung und Überreichung der Confessio Augustana an den Kaiser, in: MBW.T 4,1, Nr. 939, 263f. Vgl. auch MBW 939. 395.

12 Melanchthon an Martin Luther [auf der Coburg], [Augsburg], 27. Juni [1530], in: MBW.T 4,1, Nr. 952, 269.

13 Martin Luther an Melanchthon in Augsburg, [Veste Coburg], 29. Juni [1530], in: MBW.T 4,1, Nr. 946, 289,19-290,22.

14 Vgl. Timothy J. Wengert, Philip Melanchthon's Last Word to Cardinal Lorenzo Campeggio, Papal Legate at the 1530 Diet of Augsburg, in: Irene Dingel u.a. (Hg.), Philip Melanchthon. Theologian in Classroom, Confession, and Controversy, Göttingen 2012 (Refo500 Academic Studies 7), 79-103.

15 Vgl. Melanchthon an Kardinal Lorenzo Campeggio in Augsburg, [Augsburg, 4.] Juli 1530, zitiert nach MBW 952, 399. Ego igitur, cum existimarem reverendissimam dominationem tuam in ista sapientia quam maxime a violentis consiliis abhorrere, duxi ad reverendissimam dominationem tuam scribendum esse, ut et nos intelligeret unice cupidos esse pacis atque concordiae neque detrectare ullam tolerabilem faciundae pacis conditionem. Dogma nullum habemus diversum ab ecclesia Romana. Multos etiam repressimus, quod pernitiosa dogmata serere conati sunt, cuius res extant publica testimonia. Parati sumus obedire ecclesiae Romanae, modo ut illa sua clementia, qua semper erga omnes gentes usa est, pauca quaedam vel dissimule vel relaxet, quae iam mutare, ne quidem si velimus, queamus. (MBW.T 4,1, Nr. 952, 324,14-23). Levis quaedam dissimilitudo rituum est, quae videtur obsistere concordiae. Sed ipsi canones fatentur ecclesiae concordiam in huiusmodi rituum dissimilitudine retineri posse. (a.a.0., 325,26-326,39). 
Priesterehe sowie eine über die Messe zu erzielende Verständigung eine Eintracht beider Seiten herzustellen sei. Dies zeigt, wie fast schon verzweifelt man darum bemüht war, die Differenzen herunterzuspielen und wie unermüdlich man darum rang, keine Fronten zu schaffen, zumal sich die Aussicht auf ein Übereinkommen seit der Ankunft des Kaisers erheblich verschlechtert hatte. Denn mit dem Verbot reformatorischer Predigt und der Aufforderung an die Evangelischen, an der Fronleichnamsprozession teilzunehmen, hatte Kaiser Karl V., der am 15. Juni in Augsburg eingetroffen war, sogleich ein klares antireformatorisches Zeichen gesetzt. ${ }^{16}$ Die weiteren Ereignisse des Reichstags erwiesen, dass sich weder in der Lehre noch in den Riten die Differenzen überbrücken ließen. Die von den Kursachsen und weiteren sieben Fürsten sowie zwei Reichsstädten unterzeichnete Apologia bzw. Defensio aber machte deutlich, dass man die reformatorische Lehre keineswegs als Abweichung von der alten Kirche verstanden wissen wollte, zumal man die Konformität der in 21 Artikeln ausgeführten Lehre mit der Heiligen Schrift als Ausweis wahrer Katholizität begriff. Auch der Rückbezug auf die konziliaren Entscheidungen der Alten Kirche in Nizäa zu Trinitätslehre und Christologie sowie die Selbsteinordnung in eine lange kirchlich-theologische Tradition diente dem Aufweis der Katholizität und fungierte als verbindendes Element. Deutlicher noch wurde in der Vorrede der dezidierte Wille zur Einigkeit hervorgehoben und in diesem Zusammenhang der allen gemeinsame Ruf nach einem anzuberaumenden Konzil aufgenommen. Der zum Ausdruck gebrachte Wille zur Solidarität mit der alten Kirche stieß jedoch bei den Kernstücken der reformatorischen Lehre und Praxis an seine Grenzen: bei der Rechtfertigungslehre, der Sakramentenlehre und -praxis sowie der Infragestellung der kirchlichen Hierarchie. Dadurch aber, dass man die reformatorische Kritik an der Messe und am Papsttum an das Ende der Apologia verwies, versuchte man auch dies zu entschärfen. Überhaupt reduzierte die Confessio Augustana die Differenzen in Riten und Ordnung der Kirche auf einige wenige. Die von den reformatorisch Gesinnten vorgenommenen Änderungen wurden durch die Begründung gerechtfertigt, dass man lediglich missbräuchliche Verwendungen abgeschafft habe. ${ }^{17}$

Melanchthon und die Wittenberger Theologen sahen also das in ihrer Apologia und Defensio niedergelegte Bekenntnis zunächst und vorrangig noch als

16 Vgl. Nicole Kuropka, Philipp Melanchthon. Wissenschaft und Gesellschaft. Ein Gelehrter im Dienst der Kirche (1526 - 1532), Tübingen 2002 (Spätmittelalter und Reformation NR 21), 219-223. 17 Vgl. zur Erstellung der Confessio Augustana und ihrer Apologie Bernhard Lohse, Dogma und Bekenntnis in der Reformation. Von Luther bis zum Konkordienbuch, in: Handbuch der Dogmenund Theologiegeschichte, hg.v. Carl Andresen, Bd. 2: Die Lehrentwicklung im Rahmen der Konfessionalität, Göttingen 1984, 1-164, bes. 81-94. Außerdem Wilhelm Maurer, Historischer Kommentar zur Confessio Augustana, Bd. 1, Gütersloh 21979, Bd. 2, Gütersloh 1978. 
Weg zu einem wiederzugewinnenden bzw. neu zu etablierenden Konsens auf der Grundlage der Heiligen Schrift. Die Confessio Augustana enthielt deshalb in ihren ersten 21 Artikeln das, was man zeitgenössisch in anderen, aber vergleichbaren Kontexten als analogia fidei ${ }^{18}$ bezeichnete. Dazu knüpfte sie an die dogmatischen Entscheidungen der Alten Kirche an und stellte ihre eigenen Lehraussagen - unter Betonung des beiden Seiten Gemeinsamen - in diesen Horizont. Auch in der strukturellen Anlage des Textes und der Übernahme erprobter rhetorischer Formen spiegelte sich das Bemühen, Einhelligkeit und nicht etwa Abweichung zu demonstrieren. Denn die Confessio Augustana übernahm die Methode, Glauben und Lehre in Form von Affirmativa und Negativa zu formulieren, um auch über die Verwerfungen der klassischen, altkirchlichen Häresien die „Katholizität“ ihrer Bekenner zu belegen. ${ }^{19}$ Der Akzent lag eindeutig auf der Übereinstimmung der hier entfalteten Lehre mit der Heiligen Schrift und der ecclesia Romana, freilich nur soweit wie auch deren Lehren aus der Heiligen Schrift zu erheben seien. ${ }^{20}$

\section{Repetitio et Interpretatio - die Confessio Augustana als Knotenpunkt eines theologischen, politischen und rechtlichen Referenzsystems}

Die Confessio Augustana gewann als Bekenntnis einflussreicher Reichsfürsten und Reichsstädte rasch politische Relevanz. Zu den Unterzeichnern gehörten zum Teil jene Stände, die bereits 1529 die Speyerer Protestation verantwortet hatten. Mit Kurfürst Johann von Sachsen, Markgraf Georg von Brandenburg-Ansbach, Herzog Ernst von Braunschweig-Lüneburg, Landgraf Philipp von Hessen, Johann Friedrich von Sachsen, Franz von Braunschweig-Lüneburg, dem Bruder Ernsts,

18 Zum Ursprung des Begriffs der analogia fidei vgl. Röm. 12,6, außerdem zu seiner Bedeutung und Anwendung: Bernhard Gertz, Glaubenswelt als Analogie. Die theologische Analogie-Lehre Erich Przywaras und ihr Ort in der Auseinandersetzung um die analogia fidei, Düsseldorf 1969 (Themen und Thesen der Theologie), 53-62, hier bes. 72 .

19 Vgl. dazu Hans-Werner Gensichen, Damnamus. Die Verwerfung von Irrlehre bei Luther und im Luthertum des 16. Jahrhunderts, Berlin 1955 (Arbeiten zur Geschichte und Theologie des Luthertums 1), bes. 11-28, 65-84.

20 Vgl. zum Vorangegangenen und Folgenden auch meine analogen Ausführungen in Irene Dingel, Bekenntnis und Geschichte. Funktion und Entwicklung des reformatorischen Bekenntnisses im 16. Jahrhundert, in: Johanna Loehr (Hg.), Dona Melanchthoniana. Festgabe für Heinz Scheible zum 70. Geburtstag, Stuttgart-Bad Cannstatt ${ }^{22005, ~ 61-81 . ~}$ 
und Fürst Wolfgang von Anhalt, außerdem Nürnberg und Reutlingen waren es acht Fürsten und zwei freie Reichsstädte, die sich zur Confessio Augustana bekannten. Dieser Kreis wurde im Laufe der Zeit immer größer. Als Rechenschaft über Glauben und Lehre der protestierenden Stände des Reichs gewann die Confessio Augustana im Zuge der folgenden Entwicklungen immer mehr die Funktion eines nicht nur theologisch, sondern auch politisch und rechtlich relevanten Dokuments. ${ }^{21}$ Denn sie wurde die Bekenntnisgrundlage des unmittelbar nach dem Augsburger Reichstag geschlossenen Schmalkaldischen Bundes, ${ }^{22}$ der als Defensivbündnis der Abwehr solcher Angriffe dienen sollte, die aus Gründen abweichender Religion und damit verbundener Reformmaßnahmen von kaiserlicher Seite erfolgen könnten. Die damaligen Bündnispartner - unter ihnen Kurfürst Johann von Sachsen, Landgraf Philipp von Hessen, Herzog Ernst von Braunschweig-Lüneburg, Fürst Wolfgang von Anhalt, zwei Grafen von Mansfeld waren zum Teil mit den Unterzeichnern der Confessio Augustana identisch. ${ }^{23}$ Eine Aufnahme weiterer Mitglieder in den Bund erfolgte erst nach Annahme des Bekenntnisses. Einheit im Bekenntnis galt als Voraussetzung für das politische Bündnis, was zur Folge hatte, dass theologische Konsensverhandlungen mit denjenigen, die man als Bündnispartner zu gewinnen suchte, zu führen waren. Politische Aktion und theologische Reflexion bedingten einander; zumindest waren sie eng miteinander verzahnt. Mit dieser Entwicklung ging einher, dass man das integrative Potenzial der Confessio Augustana nun allmählich antirömisch definierte, auch wenn man mit Anhängern des alten Glaubens in Gespräche eintrat. Ein Beispiel dafür sind die Verhandlungen, die in den Jahren 1534 und 1536 mit König Franz I. von Frankreich und König Heinrich VIII. von England

21 In der Forschung ist gelegentlich die besondere Bedeutung der CA als vor allem politisches Dokument hervorgehoben worden. Vgl. Bernd Moeller, Das Reich und die Kirche in der frühen Reformationszeit, in: Bernhard Lohse/Otto-Hermann Pesch (Hg.), Das „Augsburger Bekenntnis“ von 1530 - damals und heute, München 1980, 17-31, bes. 28-30. Vgl. auch Matthias Kroeger, Das Augsburger Bekenntnis in seiner Sozial- und Wirkungsgeschichte, in a.a.0., 99-122, bes. 99-106. Demgegenüber ist festzuhalten, dass das theologische Potenzial, das die Confessio Augustana in Konzeption wie Wirkung entfaltete, so groß war wie keines der anderen Bekenntnisse des Protestantismus.

22 Vom 22. bis 31. Dezember 1530 versammelten sich die evangelischen Stände des ober-, mittelund niederdeutschen Raums in Schmalkalden, um über ein Verteidigungsbündnis zu beraten. Der Vertrag dieses Defensivbündnisses wurde am 27. Februar 1531 geschlossen. Vgl. Gabriele HaugMoritz, Der Schmalkaldische Bund (1530 - 1541/42). Eine verfassungsgeschichtliche Fallstudie zu den genossenschaftlichen Strukturelementen der politischen Ordnung des Heiligen Römischen Reiches deutscher Nation, Leinfelden-Echterdingen 2002 (Schriften zur südwestdeutschen Landeskunde 44), 1-24.

23 Vgl. Irene Dingel, Reformation. Zentren - Akteure - Ereignisse, Göttingen 2016, $192 \mathrm{f}$. 
geführt wurden. Auch bei diesen Konkordienbemühungen über die Grenzen des Reichs hinweg diente die Confessio Augustana als Grundlage. ${ }^{24}$ Die Annäherung des französischen und des englischen Königs an die Stände des Schmalkaldischen Bundes war zwar durch verschiedene politische Gründe motiviert, wie zum Beispiel die machtpolitisch antihabsburgische und zugleich humanistische Einstellung des französischen Monarchen und die Ehescheidungsangelegenheit des englischen Königs, die ihn aus verwandtschaftlichen Gründen in Gegensatz zu Habsburg brachte. ${ }^{25}$ Aber Melanchthon, der die Verhandlungen mit den französischen und englischen Gesandten führte, sah in dieser Annäherung der ausländischen Mächte an die Schmalkaldener eine reale Chance zur Wiederherstellung der Einheit der Kirche unter gleichzeitiger Beseitigung der gröbsten Missbräuche auf dem Boden einer nun auszuhandelnden theologischen Concordia. Dafür zog er die Confessio Augustana als Grundlage heran, deren Lehraussagen er an den jeweils unterschiedlichen religionspolitischen Situationen in Frankreich und England ausrichtete, jedoch unter strikter Wahrung der genuin reformatorischen Inhalte: der Lehre von der Rechtfertigung sola gratia mit deren Auswirkung auf das Abendmahlsverständnis. Das mit Guillaume du Bellay, Seigneur de Langey, ausgehandelte Consilium ad Gallos von 1534 und die mit den englischen Legaten Robert Barnes, Edward Fox (Bischof von Hereford) und Nicholas Heath (Archidiakon von Canterbury) ${ }^{26}$ im Jahre 1536 verhandelten Wittenberger Artikel wiederholten daher die Hauptaussagen des Augsburger Bekenntnisses und passten sie in die jeweiligen landesspezifisch-kirchlichen Kontexte ein. Die Konsensdokumente betonten das Gemeinsame bei gleichzeitiger Thematisierung reformatorischer Anliegen, teils unter Inanspruchnahme der gängigen altgläubigen Begrifflichkeiten. ${ }^{27}$ Allein die Lehre von der Rechtfertigung sola gratia und sola fide, die bereits in der Apologie der Confessio Augustana die Funktion eines nicht aufgebbaren, grundlegenden Kriteriums erhalten hatte, war, einschließlich ihrer Auswirkungen auf das Abendmahlsverständnis, jeglichem Kompromiss entzogen. Eigentlich waren also alle Voraussetzungen für den Beitritt

24 Vgl. zum Folgenden Irene Dingel, Melanchthon und Westeuropa, in: Günther Wartenberg/ Matthias Zentner (Hg.), Philipp Melanchthon als Politiker zwischen Reich, Reichsständen und Konfessionsparteien. Tagungsband der Wissenschaftlichen Tagung aus Anlaß des 500. Geburtstages Philipp Melanchthons, Wittenberg 1998 (Themata Leucoreana), 105-122 = dies., Melanchthon's Paraphrases of the Augsburg Confession, 1534 and 1536, in the Service of the Smalcald League, in: Dingel u. a., Philip Melanchthon, 104-122.

25 Katharina von Aragon, die Gemahlin Heinrichs VIII., war eine Tante Kaiser Karls V.

26 Zur Charakterisierung der Personen und ihrer Einschätzung durch Luther und Melanchthon vgl. Friedrich Prüser, England und die Schmalkaldener 1535-1540, Leipzig 1929 (QFRG 11), 42- 44. 27 Zum Beispiel in der Rede vom peccatum originale oder der iustificatio, die schon bei Augustin bzw. in der mittelalterlichen Scholastik üblich waren. 
der beiden Mächte zum Schmalkaldischen Bund geschaffen. Aber weder das Consilium ad Gallos noch die Wittenberger Artikel wurden letzten Endes ratifiziert.

Demgegenüber verliefen die Verhandlungen mit den Oberdeutschen unter der Führung Martin Bucers, die in die Wittenberger Konkordie von 1536 mündeten, sowohl theologisch als auch in ihrer langfristigen politischen Wirkung einigermaßen erfolgreich. Hier ging es um einen innerprotestantischen Konsens, in dem die Confessio Augustana sowohl theologisch als auch politisch als Referenzbekenntnis eine Rolle spielte, auch wenn dies nicht explizit diskutiert wurde. Im Mittelpunkt stand vielmehr ausschließlich die reformatorisch unterschiedlich ausgelegte Abendmahlslehre, die das reformatorische Lager nicht nur theologisch spaltete, sondern auch politischen Bündnissen im Wege stand. Der Wittenberger Konkordie gelang es, die innerprotestantischen Differenzen in der Abendmahlslehre mithilfe des Begriffs der manducatio indignorum auf der Grundlage einer nicht näher definierten unio sacramentalis von Leib und Blut Christi mit den Abendmahlselementen Brot und Wein dissimulativ zu überbrücken und die so konturierte Abendmahlslehre wenigstens oberflächlich konsensfähig zu machen. ${ }^{28}$ Zwar verstanden beide Seiten, Martin Luther und Martin Bucer, diesen in der Konkordie formulierten Konsens weiterhin jeweils im Horizont ihrer eigenen spezifischen Theologie, was von Seiten Luthers in den Schmalkaldischen Artikeln von 1537 wenig später einen präzisen Ausdruck fand..$^{29}$ Und auch Bucer und die Oberdeutschen blieben bei ihrer Lehre. Aber auf der Basis der Wittenberger Konkordie war ein Bekenntnis der Oberdeutschen zur Confessio Augustana und deren Apologie und damit ihre Aufnahme in den Schmalkaldischen Bund möglich geworden. Dem damit erreichten theologischen Konsens trug Melanchthon insofern Rechnung als er den Abendmahlsartikel der Confessio Augustana (Art. 10) konsequenterweise neu formulierte: In der sogenannten secunda variata von 1540 verzichtete er auf die Aussage der Anwesenheit von Leib und Blut Christi unter der Gestalt der Elemente Brot und Wein sowie auf die Verwerfung gegenteiliger Lehre und sprach lediglich davon, dass im Abendmahlsvollzug Leib und Blut Christi mit Brot und Wein dargereicht würden. ${ }^{30}$ In dieser Fassung, die Melanchthon für das von Kaiser Karl V. einberufene Religionsgespräch von Hagenau, Worms und Regensburg 1540/41 erstellte, wurde die Confessio Augustana sodann zur Disputationsgrundlage zwischen Vertretern der römischen Kirche und den Evangelischen. Hier zeigte sich noch einmal der auf Verständigung mit der römischen Kirche ausgerichtete Charakter des Bekenntnisses bei gleichzeitiger Betonung

28 Vgl. Bucer-DS 6,1, 122, die Termini Technici 122,8 und 122,3.

29 Die Schmalkaldischen Artikel, bearb. v. Klaus Breuer/Hans-Otto Schneider, in: BSELK, 713785. Vgl. bes. $766-769$.

30 Vgl. BSELK, QuM I, 127,25-27. 
dessen, was sich innerreformatorisch als integrativ und theologisch als unaufgebbar erwiesen hatte. ${ }^{31}$ Denn neben dem Artikel 10 (Vom Abendmahl) hatte Melanchthon auch den Artikel 4 (Von der Rechtfertigung), Artikel 5 (Vom Predigtamt), Artikel 6 (Vom neuen Gehorsam) und Artikel 20 (Von guten Werken) einer Überarbeitung unterzogen. ${ }^{32}$ Diese Modifikationen bzw. teils beachtlichen Erweiterungen dienten dem Zweck, ein Gespräch und wenn möglich eine Einigung auf der Grundlage der Confessio Augustana zu ermöglichen. An den Artikeln über die Rechtfertigung (4) und über gute Werke (20) wurde allerdings zugleich deutlich, wie weit sich die reformatorische Bekenntnisidentität bereits gefestigt hatte. Denn der ehemals knappe Artikel 4 war in der Variata zu einer ausführlichen Entfaltung der reformatorischen Rechtfertigungslehre ausgestaltet, beeinflusst durch die Loci Melanchthons von 1535 (secunda aetas). ${ }^{33}$ Dem stand in Artikel 20 eine ausführliche Abhandlung über Glauben und Werke zur Seite, die gleichzeitig signalisierte, das Gespräch mit der altgläubigen Position, die den Stellenwert des menschlichen Einsatzes für die Rechtfertigung festhielt, nicht abbrechen zu wollen. Dementsprechend setzte Artikel 20 gezielt damit ein, Vorwürfe der altgläubigen Gegner aufzugreifen und deutlich zu machen, dass man die guten Werke doch keineswegs abschaffen, sie aber vom rechtfertigenden Glauben her verstanden wissen wolle. „Es müssen nämlich in der Kirche beide Lehren vorhanden sein“, so führte Melanchthon aus, sowohl „das Evangelium vom Glauben zur Aufrichtung und Tröstung der Gewissen, als auch die Lehre, welches wahrhaft gute Werke und welches die wirklichen Gottesdienste sind“. ${ }^{34}$ Die Rechenschaft über den reformatorischen Glauben und die eigene Standortbestimmung, die hier selbstbewusst formuliert sind, blieben also ausgerichtet auf die Möglichkeit einer Annäherung in Lehre und Leben. Dass dies nicht gelang, zeigt, wie sehr die Dynamik des Religionsgesprächs und seiner Verhandlungen dazu beitrug, dass sich beide Seiten jener bekenntnismäßigen Differenzen bewusst wurden, die die sich formierenden Konfessionen langfristig trennten.

31 Vgl. dazu Irene Dingel, Von der Wittenberger Reformation zum Luthertum. Konfessionelle Transformationen, in: Wolfgang Thönissen/Josef Freitag/Augustinus Sander (Hg.), Luther: Katholizität \& Reform. Wurzeln - Wege - Wirkungen, Paderborn/Leipzig 2016, 239-260, bes. 243 246.

32 Vgl. BSELK, QuM I, 124f., 125f., 126, 131-138.

33 Vgl. Bernhard Lohse, Art. Augsburger Bekenntnis I, in: TRE 4 (1979), 616-628, bes. 626. Vgl. Philipp Melanchthon, Loci communes theologici (1535): De gratia et de iustificatione, in: CR 21, $420-428$.

34 Das Augsburger Bekenntnis in der revidierten Fassung des Jahres 1540 (Confessio Augustana Variata), übers. v. Wilhelm H. Neuser, Texte, Dokumente 2, Speyer 1990, 21. Vgl. BSELK, QuM I, 132,11-13. 
Zugleich wurde die unermüdlich als Grundlage theologischer Konsensverhandlungen eingesetzte Confessio Augustana zur Signatur und zum Erkennungszeichen einer nicht nur theologisch geeinten, sondern auch politisch starken, reformatorisch gesinnten Gruppe. Dadurch dass das Bekenntnis zu einer konstitutiven Grundlage des 1531 gegründeten Schmalkaldischen Bundes geworden war und immer mehr Unterzeichner gewann, rückte es in den Rang eines öffentlich und politisch relevanten Dokuments auf. Das erklärt, warum die Confessio Augustana fast von Beginn an als rechtlicher Bezugspunkt der Waffenstillstände bzw. Friedensschlüsse fungierte, die bis zum Augsburger Religionsfrieden von 1555 zwischen dem Kaiser und den reformatorisch gesinnten Ständen abgeschlossen wurden. Der erste war der Nürnberger Anstand von 1532, der einen Waffenstillstand und das Aussetzen der laufenden Kammergerichtsprozesse garantierte sowie gewaltsame Aktionen gegen die reformatorischen Stände untersagte, damit diese dem Kaiser die dringend benötigte Unterstützung gegen das vor den Grenzen des Reichs lagernde riesige osmanische Herr unter Sultan Suleiman I. zusagten. ${ }^{35}$ Der im April 1539 geschlossene Frankfurter Anstand hatte dasselbe Ziel, nämlich der kaiserlichen Seite die Unterstützung der reformatorischen, sich unter der Confessio Augustana und im Schmalkaldischen Bund zusammengeschlossenen Stände zu sichern. Der Frankfurter Anstand weitete den in Nürnberg geschlossenen Waffenstillstand auf alle seit 1532 hinzugekommenen Anhänger des Augsburger Bekenntnisses aus und sicherte ihnen für 15 Monate Schutz vor kaiserlichem Zugriff und Maßnahmen des Reichskammergerichts zu, sofern sie ihrerseits niemanden mehr in den Schmalkaldischen Bund aufnahmen und „Türkenhilfe“ leisteten. Hier ist das erste Mal von den „Augsburger Konfessionsverwandten" die Rede. Nur auf sie bezog sich die im Frankfurter Anstand gewährte, zeitlich befristete rechtliche Duldung. ${ }^{36}$ Der Augsburger Religionsfrie-

35 Vgl. den Nürnberger Anstand (24. Juli 1532), bearb. v. Andreas Zecherle, in: Religiöse Friedenswahrung und Friedensstiftung in Europa (1500-1800): Digitale Quellenedition frühneuzeitlicher Religionsfrieden, hg.v. Irene Dingel, bearb. v. Christopher Voigt-Goy u.a., http://tuedi tions.ulb.tu-darmstadt.de/e000001/ (31.05.2021).

36 Vgl. den Frankfurter Anstand (19. April 1539), bearb.v. Andreas Zecherle, in: Dingel, Religiöse Friedenswahrung und Friedensstiftung, http://tueditions.ulb.tu-darmstadt.de/e000001/ (31.05. 2021). Vgl. auch Irene Dingel, ,... das Recht haben, bei Religion, Glauben, Kirchengebräuchen in Frieden zu bleiben“. Religionsfrieden in der Frühen Neuzeit, in: dies. u. a. (Hg.), Theatrum Belli Theatrum Pacis. Konflikte und Konfliktregelungen im frühneuzeitlichen Europa. Festschrift für Heinz Duchhardt zu seinem 75. Geburtstag, Göttingen 2018 (Veröffentlichungen des Instituts für europäische Geschichte Mainz, Beiheft 124), 73-89, bes. 78-82. 
den von 1555 griff diese Bezeichnung später auf. ${ }^{37}$ Die Adressaten der in Augsburg 1555 formulierten Religionsfriedensregelungen waren also nicht - wie man in der Literatur oft liest - die Lutheraner, sondern die Augsburger Konfessionsverwandten, d.h. die sich zur Confessio Augustana bekennenden Stände, wobei vorerst unklar blieb, welche Fassung des Augsburger Bekenntnisses als maßgeblich zu gelten hatte: diejenige von 1530 oder die damals allseits im Gebrauch stehende Variata von 1540. Die Confessio Augustana war also zum Knotenpunkt eines sowohl theologischen, als auch politischen und rechtlichen Bezugssystems geworden. Der Rekurs auf dieses Bekenntnis markierte nicht nur eine besondere theologische Option, sondern auch ein politisches Bündnisgeflecht und einen religionsrechtlichen Schutzraum.

\section{Repetitio et Interpretatio - die Confessio Augustana im Spannungsfeld konfessioneller Konsolidierung}

Die im Kräftespiel von Kaiser und Fürsten verlaufenden Entwicklungen hatten wie oben dargelegt - die Confessio Augustana als Bekenntnisgrundlage des 1531 geschlossenen Schmalkaldischen Bundes und als Bezugspunkt der seit 1532 vereinbarten verschiedenen Friedstände in die Funktion eines politischen und rechtlichen Dokuments einrücken lassen. Dies war aber keineswegs der ursprüngliche Zweck des Bekenntnisses, sondern eher eine sekundäre Wirkung, die sich aus der Verschränkung von Religion und Reichspolitik ergeben hatte. Hauptanliegen der Confessio Augustana blieb die Rechenschaft über Glauben und Lehre der protestierenden Stände, gekoppelt mit dem Versuch, auch nach dem immer wieder erneuerten Wormser Edikt den Weg zu einem Konsens mit der römischen Kirche offen zu halten. Dies änderte sich mit dem Erlass des kaiserlichen Interims im Jahre 1548 nach der Niederlage des Schmalkaldischen Bundes im Krieg mit Karl V. und seinen Verbündeten. Denn das Interim zielte darauf, die Reformation weitestgehend rückgängig zu machen. Es enthielt deshalb lediglich das Zugeständnis von Laienkelch und Priesterehe, nicht aber Konvergenzformulierungen in der Lehre. ${ }^{38}$ Diesem Religionsgesetz versuchte die Leipziger Land-

37 Vgl. den Augsburger Religionsfrieden (25. September 1555), bearb. v. Alexandra SchäferGriebel, in: Dingel, Religiöse Friedenswahrung und Friedensstiftung, http://tueditions.ulb.tudarmstadt.de/e000001/ (31.05.2021).

38 Vgl. Das Augsburger Interim von 1548. Nach den Reichstagsakten deutsch und lateinisch, hg.v. Joachim Mehlhausen, Neukirchen-Vluyn ${ }^{2} 1996$ (TGET 3), hier bes. 142. 
tagsvorlage, an der Melanchthon und die Wittenberger Theologen mitgearbeitet hatten, eine Alternative entgegenzusetzen, indem sie die grundlegenden Artikel reformatorischer Lehre beibehielt und mit altgläubigen Zeremonien zu kombinieren versuchte. Zwar wurde sie nie rechtskräftig, aber dadurch, dass sie Matthias Flacius Illyricus und Nikolaus Gallus als Leipziger Interim mit polemischen Kommentaren versehen in die Öffentlichkeit brachten, wurde sie zum Auslöser heftiger innerprotestantischer Kontroversen. ${ }^{39}$ Hinter all den theologischen Auseinandersetzungen aber stand im Grunde das aufrichtige Bemühen darum, das Erbe der Wittenberger Reformation möglichst getreu an die nachfolgenden Generationen weiterzugeben. Die nun entstehenden Lehrformulierungen, Konsensdokumente oder Bekenntnisse verstanden sich daher meist als Wiederholung bzw. präzisierende Auslegung der Confessio Augustana. ${ }^{40}$ Das Augsburger Bekenntnis wurde dabei zu einer Lehrautorität, die normatives Ansehen gewann und an der sich konfessionelle Identitäten bildeten. Es wurde zugleich zu einer Referenz, auf die man sich zur Legitimierung der eigenen Bekenntnisidentität zurückbezog.

Eine solche konfessionell aufgeladene Inanspruchnahme der Confessio $\mathrm{Au}$ gustana zeigt sich prominent in dem Magdeburger Bekenntnis von 1550, das federführend von Nikolaus Gallus und Nikolaus von Amsdorf ausgearbeitet wurde. ${ }^{41}$ Beide gehörten zusammen mit Matthias Flacius u.a. zu jenen bekenntnisbewussten Lutheranhängern, die den im Leipziger Interim vorliegenden Versuch Melanchthons, der Reformation in Kursachen in der herrschenden religionspolitischen Krise eine Überlebenschance $\mathrm{zu}$ sichern, missbilligten. In ihren Augen hatte Melanchthon, indem er sich auf diesen Weg begab, seine eigenen Bekenntnisprinzipien verlassen, und dies zu einem Zeitpunkt, zu dem nach Ansicht dieser als Gnesiolutheraner bezeichneten Gruppe ${ }^{42}$ - ein klares Bekenntnis zur Wahrheit gefordert gewesen wäre. Die Theologen um Amsdorf, Gallus und Flacius, die in der Stadt Magdeburg Zuflucht gefunden hatten, veröf-

39 Vgl. Der Theologen Bedenken oder Beschluss des Landtages zu Leipzig 1548 (Leipziger Interim), bearb. v. Hans-Otto Schneider, in: Der Adiaphoristische Streit (1548-1560), hg.v. Irene Dingel, Göttingen 2012 (Controversia et Confessio 2), 354-441.

40 Vgl. zu dem Folgenden auch Dingel, Bekenntnis und Geschichte, 75-81.

41 Vgl. Magdeburger Bekenntnis, lateinisch und deutsch (Magdeburg 1550), bearb. v. Hans-Otto Schneider, in: Dingel, Der Adiaphoristische Streit, 442-629.

42 Vgl. dazu Rudolf Keller, Art. Gnesiolutheraner, in: TRE 13 (1984), 512-519. Zu den konfessionsbezogenen Gruppenbezeichnungen und ihre Problematik vgl. Irene Dingel, Concordia controversa. Die öffentlichen Diskussionen um das lutherische Konkordienwerk am Ende des 16. Jahrhunderts, Gütersloh 1996 (Quellen und Forschungen zur Reformationsgeschichte 63), 17-19 und dies., Historische Einleitung, in: Reaktionen auf das Augsburger Interim, hg.v. ders., Göttingen 2010 (Controversia et Confessio 1), bes. 10-12. 
fentlichten daher mit dem Magdeburger Bekenntnis ein Dokument, das als Wiederholung der Confessio Augustana die Rechtmäßigkeit ihrer Lehre belegen und ihren Widerstand gegen den Kaiser legitimieren sollte. Dies brachte der dem Bekenntnis vorangestellte „Kurtze begriff“ deutlich zum Ausdruck: „Erstlich: das vnsere Kirchen allhie mit warer Christlichen Religion vnd Gottesdienst versehen sein. Des thun wir allhie vnsere bekentnis fu(e)r vns vnd vnsere gantze Christliche Gemeine, welche sich zeucht auff die Artickel der Augspurgischen Confession im ersten teil dieses Buchs. “43 Nun ging es nicht mehr um Konsensstiftung und Integration, sondern um eine dezidierte Abgrenzung der rechten Lehre von der falschen. Falsche Lehre aber erblickte man nicht nur auf der Seite der römischen Kirche, sondern auch bei Melanchthon und seinen Anhängern, die bereit waren, sich im Grenzbereich des Indifferenten politischen Zwängen anzupassen, indem sie die altgläubigen Riten als Adiaphora zuließen. ${ }^{44}$ Diese Haltung stieß bei den Widerständlern und Autoren des Magdeburger Bekenntnisses auf Unverständnis. Sie kultivierten dagegen eine ganzheitliche Bekenntnishaltung, die Lehre und kirchlich-liturgische Praxis gerade in casu confessionis et scandali ${ }^{45}$ kompromisslos aufeinander bezog und zusammenband. Dieses Beispiel zeigt, dass hier die Funktion des Bekenntnisses auf der identitären Vergewisserung lag, für die man die Lehre der Confessio Augustana normativ zugrunde legte. Zugleich wird erkennbar, welch hohe theologische Autorität die Confessio Augustana inzwischen erhalten hatte. Die Magdeburger Bekenner sahen nämlich im Interim eine Unterdrückung von „namen vnd [...] ansehen der Augspurgischen Confession vnd lere“46, deren Inhalt man nicht nur als analogia fidei wertete, sondern nun auch mit der Theologie Martin Luthers identifizierte, den man als endzeitlichen Propheten stilisierte und dessen Lehre man um so mehr Gewicht beimaß. ${ }^{47}$ Aber mehr noch: eine Verleugnung der Confessio Augustana kam in ihren Augen einer Verleugnung Christi gleich. ${ }^{48} \mathrm{Um}$ dem Nachdruck zu verleihen und um nachzu-

43 Magdeburger Bekenntnis, deutsch (1550), in: Dingel, Der Adiaphoristische Streit, 459,15-19. 44 Vgl. dazu: Dingel, Der Adiaphoristische Streit, 471,2-6: „Dargegen wollen wir zuweilen setzen, wo von diesem reinen einhelligen verstand Christlicher Lere vnd bekentnis abgewichen sind Papisten, Jnterimisten vnd Adiaphoristen, desgleichen Widerteuffer, Sacramentirer vnd was mehr irriger Geister sind, von welchen allen wir vns mit Lere, bekentnis vnd mit wercken gentzlich absundern.“

$45 \mathrm{Zu}$ diesem Begriff bei Flacius vgl. Hans Christoph von Hase, Die Gestalt der Kirche Luthers. Der casus confessionis im Kampf des Matthias Flacius gegen das Interim von 1548, Göttingen 1940.

46 Magdeburger Bekenntnis, 467,22f.

47 Vgl. Magdeburger Bekenntnis, $465 \mathrm{f}$.

48 Vgl. Magdeburger Bekenntnis, 467,28-31: „Diese stu(e)ck vnd viel andere mehr, wie sie in der warheit nichts anders sind denn ein verleugkung der Augspurgischen Confession vnd mit der- 
weisen, dass das Augsburger Bekenntnis von Anfang an und in allen Disputationen und Konsensgesprächen unwiderlegt und unverändert geblieben sei, bot das Magdeburger Bekenntnis nicht nur Lehr- und Bekenntnisaussagen und eine Entfaltung der Widerstandslehre, sondern auch eine kleine Geschichte der Confessio Augustana..$^{49}$ Die Magdeburger Bekenner selbst sahen sich als „,vberbleiblinge von der Augspurgischen Confession“, deren Pflicht es sei, von dem durch Luther wiederentdeckten Evangelium und der Confessio Augustana Zeugnis abzulegen. Allen unter Verfolgung leidenden Christen sollte auf diese Weise vermittelt werden, dass dieses Bekenntnis zur Wahrheit, dessen Inhalt man in Abgrenzung von allen lehrmäßigen Pervertierungen wiederholte, keineswegs seine Geltung eingebüßt habe..$^{50}$

Dass die strengen Lutheranhänger für sich in Anspruch nahmen, die einzigen und wahren „vberbleiblinge von der Augspurgischen Confession“ zu sein, war aber nur eine, und zwar ihre eigene Perspektive, mit der sie sich von einer vermeintlichen, nach Luthers Tod eingetretenen theologischen Fehlentwicklung distanzierten, für die sie Melanchthon verantwortlich machten. Aber auch für diesen und seine Anhänger war die Confessio Augustana zu einer normativen Autorität geworden, die, nach außen abgrenzend, evangelische Identität definierte. Allerdings versuchte Melanchthon die gegenüber dem römischen Katholizismus nun abgrenzend formulierte Identitätsaussage mit dem Bemühen um die innere Einheit und den Konsens aller Anhänger des Augsburger Bekenntnisses zu kombinieren. Bestes Beispiel dafür ist die Confessio Saxonica, auch genannt Repetitio Confessionis Augustanae, die der Wittenberger im Jahr 1551 auf Veranlassung des Kurfürsten Moritz von Sachsen zur Vorlage auf dem Konzil von Trient erstellte. ${ }^{51}$ Die Hoffnung darauf, dass dieses Konzil der Beilegung der religiösen Differenzen und der Findung eines Konsenses dienen könnte, war lange gestorben. Folgerichtig betonte Melanchthon in der Einleitung zur Confessio Saxonica mit Bezug zur gegenwärtigen Situation, dass es vor allem dann, wenn den Kirchen Irrtum und falsche Lehre zur Last gelegt werde und man ihnen vorwerfe, sich durch Spaltungen vom allgemeinen Konsens zu entfernen, nötig sei, die eigene Lehre standhaft $\mathrm{zu}$ vertreten. Necesse est interrogatos recitare doctrinam. ${ }^{52}$ Das

selben auch Christi des Herrn selbs, also nemens Babst, Bischoffe, Fu(e)rsten vnd jhr gantzer hauffe nicht anders an [...]“.

49 Vgl. Magdeburger Bekenntnis, 463-465.

50 Vgl. Magdeburger Bekenntnis, 469, das Zitat 469,15.

51 Die Confessio Saxonica ist abgedruckt in CR 28, 369-468 (lat.), 481-568 (dt.). Zur Benennung als Repetitio [...] vgl. bes. ebd., 343f. und 471-474.

52 Repetitio Confessionis Augustanae, 369. 
Bekenntnis als vera explicatio doctrina $e^{53}$ dient hier dazu, einer falschen Beurteilung durch die Gegner entgegenzuwirken und zugleich die innere Eintracht in Glauben und Lehre durch starke bekenntnismäßige Konturen zu sichern. ${ }^{54}$ Es ging jetzt um Erhalt und Stärkung des innerprotestantischen Konsenses. Den Gedanken, die römische Seite noch gewinnen zu können, hatte man längst aufgegeben. Dementsprechend positioniert die Confessio Saxonica sich und ihre Anhänger in dezidierten Kontrast zu jenem römischen Katholizismus, der sich im Konzil von Trient konsolidierte. Die Confessio Saxonica bzw. die Repetitio Confessionis Augustanae zielte darauf, die Übereinstimmung der eigenen Bekenntnisinhalte mit dem wahren consensu[s] catholicae Ecclesiae Domini nostri Iesu Christi, vnd Christlicher einigkeit ${ }^{55} \mathrm{zu}$ erweisen, d.h. mit einem catholicus consensus ecclesiae $^{56}$, der nicht nur durch die Schriften der Propheten, Apostel und Väter, sondern auch durch reformatorische Zeugnisse, besonders die Confessio Augustana, garantiert wird. ${ }^{57} \mathrm{Um}$ dies $\mathrm{zu}$ erweisen ist auch hier ein Rückblick auf die Geschichte der Reformation vorangestellt. ${ }^{58}$ Die Confessio Augustana und ihre mit der Confessio Saxonica vorliegende Wiederholung werden in die Sukzession von Wahrheitszeugen eingeordnet, die die Reformation aus ihrer Historie heraus legitimieren.

\section{Conclusio}

Die hier aufgezeigte Entwicklung der Confessio Augustana von einem vornehmlich auf theologische Integration zielenden und für Konsensverhandlungen herangezogenen Bekenntnis bis hin zu ihrer Indienstnahme als Zeugnis einer authentischen und deshalb noch unverderbten, reinen reformatorischen Lehre in $\mathrm{Ab}$ grenzung von vermeintlichen Pervertierungen vollzog sich im Kontext der jeweiligen politischen Entwicklungen. Nur in einer solchen Kontextualisierung wird deutlich, welch große Ausstrahlung die Confessio Augustana in ihren un-

53 Repetitio Confessionis Augustanae, 371.

54 Vgl. dazu auch das Vorwort der Repetitio der Augspurgischen Confession in Georg Majors Übersetzung, in: CR 28, 481.

55 Repetitio der Augspurgischen Confession, 488.

56 Repetitio der Augspurgischen Confession, 487.

57 Vgl. Repetitio Confessionis Augustanae, 376, und Repetitio der Augspurgischen Confession, 488.

58 Es handelt sich allerdings noch nicht um eine ausgearbeitete Historia der Confessio Augustana, wie sie später erstellt wurden. Vgl. Repetitio Confessionis Augustanae, 371-373, und Repetitio der Augspurgischen Confession, 482f. 
terschiedlichen Fassungen als Knotenpunkt eines weit verzweigten Referenzsystems erhalten konnte. Dabei blieb sie noch lange konfessionell offen und unbestimmt, so dass Friedrich der Fromme, Kurfürst von der Pfalz, unter Rekurs auf die von Heinrich Bullinger erstellte und in Heidelberg gedruckte Confessio Helvetica posterior versuchte, die Übereinstimmung seiner konfessionellen Option mit dem Augsburger Bekenntnis und dessen Apologie nachzuweisen, als ihm auf dem Reichstag von 1566 wegen calvinistischer Tendenzen ein Ausschluss aus dem Augsburger Religionsfrieden drohte. ${ }^{59}$

Nicht nur Melanchthon selbst, sondern auch seine Schüler, sowohl die Philippisten, d.h. jene, die sich in Melanchthons Nachfolge sahen, aber seine Theologie zugleich weiterentwickelten, als auch die Gnesiolutheraner, d.h. diejenigen, die sich in der Interimszeit von ihrem einstigen Lehrer Melanchthon abwandten und sich fortan als dezidierte Sachwalter der Theologie des verstorbenen Luther verstanden, formulierten ihre Bekenntnisse als Wiederholungen der Confessio Augustana bzw. als interpretierende Neuformulierung dieses in ihren Augen falsch ausgelegten Bekenntnisses. Alle sich hinter der jeweiligen Repetitio oder Interpretatio sammelnden Bekenner beanspruchten für sich, in der allein rechtmäßigen Nachfolge und Entwicklung der Confessio Augustana zu stehen, die inzwischen zu einer sekundären Autorität neben der Heiligen Schrift herangereift war. Angesichts dessen und im Hinblick auf die innere Zersplitterung des Protestantismus wurde es nun notwendiger denn je, $\mathrm{zu}$ einem übergreifenden reformatorischen Bekenntniskonsens zurückzufinden. Tatsächlich wurde in den sechziger Jahren des 16. Jahrhunderts ein großangelegtes Konkordienwerk in Angriff genommen, dessen Ergebnis, Konfordienformel und Konkordienbuch von 1577/80, wieder auf die Confessio Augustana invariata von 1530 rekurrierte. Der erstrebte übergreifende Bekenntniskonsens aber kam nicht zustande; das Konkordienwerk wurde Auslöser für weitere Kontroversen. ${ }^{60}$ Dadurch entwickelte sich die Confessio Augustana langfristig zu dem, was sie ursprünglich nicht sein sollte,

59 Kaiser Ferdinand I. hatte den pfälzischen Kurfürsten in einem Schreiben vom 13. Juli 1563 mitgeteilt, dass die Annahme des Heidelberger Katechismus einen Selbstausschluss aus dem Augsburger Religionsfrieden bedeute. Tatsächlich kam es zu Aktionen in Richtung auf einen Ausschluss. Dem Kurfürsten lag deshalb viel daran, die Konformität seines Landes mit dem im Reich seit dem Augsburger Religionsfrieden von 1555 geltenden Religionsrecht nachzuweisen. Dem diente das Bekenntnis Bullingers, an den sich der Kurfürst in dieser Angelegenheit gewandt hatte. Vgl. dazu genauer Endre Zsindely, Art. Confessio Helvetica Posterior, in: TRE 8 (1981), 169 173, außerdem Dingel, Concordia controversa, 101f. und dies., Bullinger und das Luthertum im Deutschen Reich, in: Emidio Campi/Peter Opitz (Hg.), Heinrich Bullinger. Life - Thought - Influence. Zurich, Aug. 25-29, 2004, International Congress Heinrich Bullinger (1504-1575), Bd. 2, Zürich 2007 (Zürcher Beiträge zur Reformationsgeschichte 24), 755-777, bes. $762 \mathrm{f}$.

60 Vgl. dazu Dingel, Concordia controversa. 
nämlich zu einem Bekenntnis im Sinne konfessioneller Abgrenzung und Identitätsbildung des Luthertums.

Diese Entwicklung und die theologisch unterschiedliche Inanspruchnahme der Confessio Augustana als Bekenntnisautorität, begünstigte - vor allem im Kontext reichspolitischer Religionsfriedensregelungen - das Entstehen einer kontrovers verlaufenden Bekenntnishistoriographie, die versuchte, die Entwicklung des Bekenntnistextes, wie sie sich an den diversen Veränderungen zeigte, zu legitimieren oder - mit Blick auf die unterdessen nicht mehr auffindbare ursprüngliche und authentische Fassung - zu delegitimieren. ${ }^{61}$ Ein Bekenntnis als Gegenstand und sogar Zentrum von Geschichtsschreibung - dies belegt nicht nur seinen Charakter als Referenzbekenntnis, sondern verstärkte auch die der Confessio Augustana beigemessene Autorität.

61 Vgl. Irene Dingel, Lutherische Bekenntnisbildung zwischen theologischer Abgrenzung und Integration, in: Lutherische Theologie und Kirche 40 (2016), 149-169, bes. $151 \mathrm{f}$. 
